\title{
Chemical "soup" clouds connection between toxins and poor health
}

\section{Toxicologist Linda Birnbaum has tried to put sound science at the center of debates over chemical regulation.}

\section{Brendan Borrell}

23 November 2012

\section{An article by Scientific American.}

From plastics to flame retardants, the ubiquitous chemicals of our daily lives have raised public health concerns like never before. Inside the Beltway, however, datacrunching scientists are often no match for industry lobbyists and corporate lawyers. The exception, no doubt, is Linda Birnbaum, the toxicologist who leads, two littleknown scientific agencies, the National Institute of Environmental Health Services (NIEHS) and the National Toxicology Program (NTP).

Last April, Birnbaum sat inside a Capitol Hill conference room packed with pokerfaced chemical industry executives ready for a showdown. The NTP had recently issued its report on carcinogens-a sort of name-and-shame list of chemicals on which no company wants to find its products. Charles Maresca of the Small Business Administration-taking a stand for the maligned styrene industry-argued that the report was "based on inaccurate scientific information" and faulty peer review.

North Carolina congressman Brad Miller (D) was unimpressed. He took the microphone and described Birnbaum's resume of more than 700 publications in public health, toxicology and environmental science. Removing his black reading glasses, he glanced at Maresca, and delivered the fatal blow with relish: "And you're a lawyer. Isn't that right?"

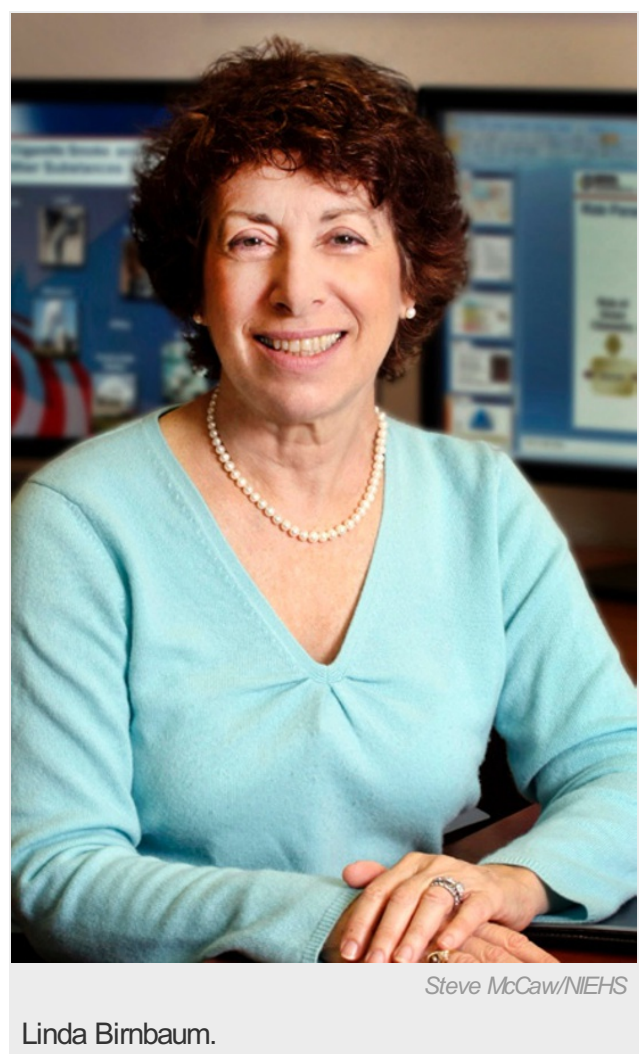

If Birnbaum got a kick out of the put-down, she didn't show it. After 33 years working as a federal scientist at both the U.S. Environmental Protection Agency and the NIEHS, Birnbaum's career is a study in the way science becomes law and the ways lobbyists subvert science. She has watched her contributions to an EPA report on dioxin sit in limbo for 20 years, she has worked to study the health impacts of types of asbestos that are not legally recognized as asbestos and she has challenged the chemical industry in her pursuit for answers about the controversial chemical bisphenol A (BPA).

Through her leadership at NIEHS and NTP over the past three years, she has pursued a broad vision of environmental health that incorporates gene-environment interactions along with the impacts of disease, diet, stress and other factors. She has also tried to make the NIEHS quick on its feet: After the 2010 BP oil spill, she initiated the Gulf Long-Term Follow-Up (GuLF) study, the first extended review of the health effects of an oil spill.

Scientific American sat down with Birnbaum in Washington, D.C., to learn more about environmental health, toxic chemistry and the politics of chemical regulation.

[An edited transcript of the interview follows.]

\section{How did you become interested in toxicology?}

When I was in eighth grade at Benjamin Franklin Junior High School in Teaneck, New Jersey, I had a science teacher who was an attractive, peppy, young blonde woman who was also the cheerleading coach. I was a cheerleader, and that positive reinforcement made it okay to like science. 
they could give me some rats and some chemicals. That's something that would never happen easily today — but they did it! I got a letter from them that said, "Please come. We'd like to talk to you." The next thing I knew, I had 40 rats in four cages and feed and bedding and everything else, along with thyroid hormone and chemicals that block thyroid hormone.

\section{They let you keep the rats at your house?}

Yeah. We had them in my basement.

\section{What did your parents say about that?}

My parents were really incredibly supportive-even when one escaped. I eventually found its body and put it in the freezer figuring l'd dissect it at some point. But my grandmother went in thinking it was a package of ground beef. She had a little bit of a fright.

\section{How much of human disease is due to environmental exposures?}

The estimates vary, and it depends on how you define environment. People often say it's about 30 percent. I think that's defining environment fairly narrowly, considering only environmental chemical exposures, but your environment includes the food you eat, the drugs you take, the psychosocial stress you're exposed to and so forth. After all, what's the difference between a drug and an environmental chemical? One you intentionally take and the other one you don't. Considering all that, I would say then the environment is much more than 30 percent.

We also know-especially from studies of identical versus fraternal twins—-that for many different diseases, genetics is not the whole story. Actually, I think it's time to stop asking, "Is this caused by genes or is this caused by the environment?" because in almost all cases, it's going to be both.

\section{Why has it been so difficult to link environmental exposures to specific health consequences?}

Nobody is exposed to one chemical at a time, right? I mean we live in a soup of chemicals and we live in a soup of exposures. Here, I'm having a lemonade. Well, it's not only lemon in here. I'm sure there's some sugar. There might be a preservative or something. I don't know what's in this. So think of all those things interacting, but when we test chemicals in the lab we tend to test them one at a time.

\section{I guess we don't consider these other types of exposures.}

Right. A high-fat diet, for example, can completely change the way your body handles chemicals. Exposure to a certain chemical may lower your ability to respond to an infection. At EPA we did a lot of studies exposing rats and mice to air pollutants and then to bacterial infections or influenza infections. Those who were exposed to pollution were more likely to die, whereas those in clean air recovered.

We see the same thing with individual kinds of chemical exposures as well. One of our NIEHS grantees, Philippe Grandjean of Harvard University, followed women during their pregnancy, and then he followed their children. So he had blood samples before they were born from the mother. He had blood samples at birth, and he continued to follow the kids. What he found is that if those children had elevated exposure to PCBs [polychlorinated biphenyls used in electronics], they were less able to mount a normal response to a vaccination.

PCBs are considered likely carcinogens, but they are also endocrine disruptors, like bisphenol A or dioxin, which is something we've heard a lot about in the media lately. What is your definition of an endocrine disruptor? An endocrine disruptor is anything that affects the synthesis of a hormone, the breakdown of a hormone or how the hormone functions. We used to think it had to bind with a hormone receptor but endocrine disruptors can perturb hormone action at other stages in the process.

\section{Why are they such a big deal?}

They're all around us, and I think they can affect us at very low levels. Our hormones control our basic homeostasis, our basic physiology. If you alter your hormone levels, you're not going to behave the same way physiologically, and that includes mentally and everything else. I think that there's growing evidence that some of the chemicals to which we are exposed are doing that to the population right now.

So we have this soup of endocrine disruptors, air pollution and other exposures, which has made epidemiology so difficult. What have you been doing at NIEHS to get to the bottom of these issues?

There are ways statistically that you can control for some of these factors, but I think we have to go beyond that and say, "Well, wait a minute. That's not real life." Maybe we need to look at whether there are interactions. In animal systems, from work that I did starting in the '80s and have continued ever since, we showed that for chemicals that have the same mechanism of action you can basically add 
up those chemicals to predict the toxicity. This is the toxic equivalency factor approach.

We've been finding with a lot of endocrine disruptors that if they impact the same health effect, such as decreased sperm production, you can just kind of add up chemicals. They may have a different mechanism, but they all affect sperm count. A lot of data show that if chemicals are estrogenic, you can add up their potency. If chemicals are anti-androgenic, you can add them up. If chemicals affect thyroid, you can add them up, too.

SCIENTIFIC Nobody has tried to look even more broadly and say, "Well, I'm gonna take all these chemicals that AMERICAN T $^{\text {TM }}$ somehow block male reproduction, and I'm also gonna add that to the chemicals that do something More from Scientific American. totally different." The way I think we're going to have to eventually get at that is through what we're calling "Tox21," which is this rapid-screening approach being developed with high-throughput screening of in vitro assays. They are cell-based assays looking at many, many, many different kinds of responses. Basically, we can screen up to 10,000 chemicals a year at 15 different dose levels for at least 70 or more different kinds of responses. We can begin now to do this with mixtures where we can make many different kinds of mixtures because we can test so many at a time.

\section{How has spending 33 years studying toxic chemicals affected your outlook on the environment?}

We do know that there are many chronic health conditions, non-communicable health conditions, which have increased too rapidly in the last 20 to 40 years. These are things like autism, ADHD and, of course, obesity and diabetes. We have identified chemicals clearly at play in the obesity epidemic. I am not in any way saying to people you can stop exercising and you don't have to watch what you eat, but the question I have is: Are we setting people up to fail because they're exposed to something that alters their ability to metabolize fats or sugars?

Overall, I'd say I'm a pragmatist. I think our air in this country is cleaner than it was, but it's not as clean as it needs to be. Our water is cleaner, but again not as clean as it needs to be. We've taken care of the really blatant environmental problems. We're concerned now about low levels of lead, not the very high levels that there used to be in our environment.-That's a good thing.

Nature | doi:10.1038/nature.2012.11881 